Published in final edited form as:

J Autism Dev Disord. 2018 June ; 48(6): 1895-1907. doi:10.1007/s10803-017-3431-7.

\title{
Parental Broad Autism Phenotype and the Language Skills of Children with Autism Spectrum Disorder
}

\author{
Michelle Flippin and \\ University of Rhode Island \\ Linda R. Watson \\ University of North Carolina, Chapel Hill
}

\begin{abstract}
Father-child and mother-child interactions were examined in order to investigate concurrent associations between three characteristics of parental broad autism phenotype (i.e., aloofness, rigidity, pragmatic language deficits), parental verbal responsiveness, and language skills of children with ASD. Results for mothers indicated that aloofness and rigidity were negatively associated with both child leads and child language skills. Maternal aloofness was also negatively correlated with verbal responsiveness to their children, but rigidity was not. Results suggest that the association between aloofness and child language are potentially mediated by maternal use of responsive verbal behaviors. Maternal pragmatic language deficits were not concurrently related to child leads or language skills. In contrast, for fathers, aloofness and rigidity were unrelated to child leads and language skills. Paternal pragmatic deficits were not associated with child language, however significant positive associations were found between paternal pragmatic language deficits and frequency of child leads. Results are discussed in reference to potential clinical implications and directions for future research.
\end{abstract}

\section{Keywords}

autism spectrum disorder; language; broad autism phenotype; father; responsiveness

\section{Introduction}

One of the earliest markers of autism spectrum disorder (ASD) is delay in language development (American Psychiatric Association, 2013). A strong predictor of language development for children with ASD is early parental verbal responsiveness. Parental verbal responsiveness refers to parents' use of language input that follows the child's lead and maps to the child's focus of attention (Landry, Smith \& Swank, 2006; Spiker, Boyce \&

Correspondence concerning this article should be addressed to Michelle Flippin, Department of Communicative Disorders, University of Rhode Island, Kingston, RI, 02881, Contact: mflippin@uri.edu.

Author Contributions. MF conceived of the study, participated in its design and coordination and drafted the manuscript; LRW participated in the study design and interpretation of the data and helped to draft the manuscript. All authors read and approved the final manuscript.

Compliance with Ethical Standards

Conflict of Interest. The authors declare that they have no conflict of interest. 
Boyce, 2002; Warren \& Brady, 2007). In two longitudinal studies, Siller and Sigman (2002, 2008) noted that mothers' use of responsive verbal strategies with preschoolers with ASD was positively correlated with joint attention and language skills over periods of one, 10 and 16 years. A strong positive relationship between maternal responsiveness and child language skills was also reported by McDuffie and Yoder (2010), who found that after controlling for child engagement, mothers' use of comments and directives that mapped the child's focus of attention (i.e., follow-in-comments and follow-in-directives) significantly predicted children's spoken vocabulary at 6-month follow-up. Haebig, McDuffie and Ellis Weismer (2013) also documented stronger one-year language outcomes for 40 toddlers and preschoolers with ASD whose parents used more responsive verbal behaviors. Finally, in a study that examined nine theoretically-motivated potential predictors of expressive language growth, Yoder, Watson and Lambert (2015) found that parent verbal responsiveness accounted for significant unique variance in expressive language gains across a 12-month time period for 87 young, initially preverbal children with ASD.

Given that these longitudinal studies show parental verbal responsiveness to be a predictor of language outcomes for children with ASD, it follows that increasing parent responsiveness is often a target of early communication intervention for children with ASD. However, results from studies of parent-implemented intervention have been mixed. For instance, three randomized control trials of parent-implemented intervention for preschoolers with ASD failed to detect main effects on child outcome measures of parent coaching on responsive strategies (Carter et al., 2011; Green et. al., 2015; Rogers et al., 2012). Nevertheless, the Carter et al. and Green et al. studies reported medium to large effects on parent outcomes, including increased parent responsivity, synchronization or interaction skills. Taken together, findings from these studies suggest that parents can be effectively taught to implement a broad range of intervention strategies. However, not all parents acquire the same level of proficiency in using targeted strategies. Moreover, parents differ in their use of these strategies over time, and some may not implement targeted strategies with sufficient intensity to affect children's long-term communication outcomes (Carter et al., 2011; Rogers et al., 2012; Schertz et al., 2013).

One possible reason for lack of main effects of parent-implemented communication intervention on child outcomes is related to the fit of treatment strategies to the preintervention characteristics of participating children. For example, Haebig and colleagues (2013) documented that parents' use of one type of responsive strategy, follow-in comments, was relatively more effective for children who began the study with lower expressive vocabulary. Thus, baseline communication level is likely one child characteristic that influences treatment outcomes. Likewise, another reason for differences in intervention outcomes may be related to the fit of treatment strategies to the pre-intervention characteristics, needs and strengths of participating parents and families. For instance, in a recent randomized controlled trial, Wetherby et al. (2014) compared effects of two 9-month, parent-implemented interventions on children with ASD. Findings showed greater improvements on child outcome measures of social communication, adaptive behaviors, and developmental level for individualized home-based parent coaching versus parent training delivered in a group setting. Thus, tailoring intervention to fit the needs of individual 
families may be more effective in improving child communication outcomes than a one-sizefits-all approach.

Although an emerging body of evidence supports the inclusion of pre-treatment child characteristics (e.g., autism severity, sensory profile, baseline cognitive and language skills) as influential variables on intervention efficacy for children with ASD, few studies have examined the potential influence of pre-treatment parent characteristics on communication outcomes for children with ASD. In a rare example, Siller, Hutman and Sigman (2013) found that mothers who initially demonstrated accepting insightfulness into their children's thoughts, feelings, and behaviors increased their synchronous responses to their young children with ASD in an intervention targeting responsive parent strategies, but mothers who were initially noninsightful did not change in responsiveness if assigned to the intervention. The Siller et al. findings illustrate the point that, because parent-implemented interventions for young children with ASD aim to improve child communication skills through changes in parent behavior, pre-intervention parental variables may also influence treatment outcomes.

One potentially influential variable in parent uptake of a parent-mediated intervention is parent gender. Despite the increased role many fathers now play in direct childcare for all children (Pleck \& Masciadrelli, 2004) and the high level of father engagement in education and learning for children with ASD (Potter, 2016), fathers have been underrepresented in ASD intervention research. However, it is estimated that two-thirds of children with ASD in the United States reside in a two-parent household (Freedman, Kalb, Zablotsky, \& Stuart, 2012) and including fathers is an important step towards effectively involving all stakeholders in ASD intervention. Moreover, specifically examining the contributions of fathers is important given that fathers of both children who are typically developing and at risk use language models that are distinct from mothers and thus may influence child language in unique ways. In fact, fathers' vocabulary use at 24 months has been shown to predict levels of child expressive language 1 year later at 36 months (Baker, VernonFeageans, \& The Family Life Project Investigators, 2015; Pancsofar \& Vernon-Feagans, 2006, 2010), whereas mothers' language did not account for a significant portion of the variance. Overall, compared to mothers, fathers tend use vocabulary that is more varied and abstract, use higher-level syntax, and are more likely to direct questions to their children (Bernstein-Ratner, 1988; Gleason, 1975; Leech, Salo, Rowe \& Cabrera, 2013; Masur \& Gleason, 1980; McLaughlin, Schultz \& White, 1980; Pancsofar \& Vernon-Feagans, 2006; Rondal, 1980; Rowe, Coker, \& Pan, 2004; Walker \& Armstrong, 1995). Paternal communication styles may be facilitative in supporting child social communication development. For example, Pelligrini, Brody \& Stoneman (1987) reported that while mothers tended to ignore their typically developing child's violations of conversational conventions, fathers used these opportunities to provide feedback to their child through repetition, requesting clarification, and modeling correct responses.

For children with ASD, who have impaired awareness of the impact of their communicative signals on others, the directive, didactic communication style of many fathers may help them to clarify their message, and increase their awareness of the impact of their communication on others. In fact, early studies reported similar patterns between fathers of children with autism and fathers of children who are typically developing, with fathers of children with 
autism also using fewer questions, labels, expansions, and prompts of child utterances and a greater percentage of directives than mothers (Konstantareas, Konstantareas, Mandel, \& Homatidis 1988; Wolchik, 1983). Although more current studies are needed to find out if these patterns between fathers and children with ASD still hold, more broadly, recent studies of fathers of both children who are typically developing and those who are at-risk for developmental problems due to socio-environmental factors show responsive fathering to be a strong predictor of positive developmental and academic outcomes including emotional regulation, cognition, and language development (Shannon, Tamis-LeMonda, London, \& Cabrera, 2002; Tamis-LeMonda, Shannon, Cabrera, \& Lamb, 2004). For instance, research with low-income families has shown that fathers who read, tell stories, and sign songs, have preschool aged children with better pre-literacy and attention skills (Baker, 2013). It may be the case that fathers' responsive verbal models are similarly associated with positive social communication outcomes for children with ASD. Thus, parent gender is one important, although often overlooked, variable that could impact the effectiveness of parent-mediated communication intervention for children with ASD. Another potentially relevant parent variable is the broad autism phenotype.

The broad autism phenotype (BAP) refers to the phenotypic expression of elevated, but diagnostically subthreshold, levels of autism-related symptoms among relatives of individuals with ASD (Folstein \& Rutter 1977). The reported prevalence of the BAP among these family members ranges from 12 to $30 \%$, and fathers have been reported to show more BAP characteristics than mothers (Bishop et al. 2004; Hurley, Losh, Parlier, Reznick \& Piven, 2007; Pickles et al., 2002). Personality, social, and language features that comprise the BAP parallel the defining behavioral characteristics of ASD, including social deficits, communication abnormalities, and stereotyped, repetitive behavior (Piven, Palmer, Jacobi, Childress \& Arndt, 1997). Specifically, researchers have defined three primary components of the BAP: (a) aloofness; (b) rigid personality; and (c) pragmatic language deficits (Hurley et. al., 2007). Aloofness is characterized by diminished interest in or enjoyment of social interaction. Rigid personality is defined as difficulty adjusting to change. Finally, pragmatic language problems refer to deficits in the social use of language, resulting in reduced effectiveness of communicative exchanges.

Emerging evidence suggests that BAP characteristics can impact the communicative effectiveness of parents during exchanges with other adults. For example, compared to parents of children with Down syndrome, parents of children with ASD were found to use less frequent eye contact in interactions with adult examiners, and fathers of children with ASD used significantly less eye contact than mothers (Ruser et al., 2007). Likewise, other researchers reported that fathers of children with ASD were more likely than fathers of typically developing children to exhibit gaze direction patterns similar to those found in ASD (Scheeren \& Stauder, 2008). In fact, Hartley, Papp, Blumenstock, Floyd and Goetz (2016) recently identified parental BAP as a vulnerability factor for families of children with ASD. Specifically, parental BAP was related to a higher overall rating of negative affect in couple interactions. Moreover, parental BAP levels moderated the impact of parents reporting a high level of child ASD symptoms and co-occurring behavior problems on same and next-day ratings of negative couple problem-solving interactions. Taken together, results of these studies suggest that the BAP may impact parent couple interactions and adult-adult 
communication. Likewise, the BAP may also influence parent-child communication. Given the strong link between the quality of early parent-child communication and later language outcomes for children with ASD, understanding any potential relationships between parental BAP and child communication skills may have important clinical implications for parentmediated interventions.

Finally, appropriately interpreting associations among parent characteristics, parent-child interaction variables, and child outcomes requires mindfulness that parents and children have bidirectional impacts on one another. The focus in parent-mediated interventions often is overwhelmingly placed on the effects that parental interaction strategies may have on children's outcomes, such as their growth in communication skills. The effects that children are having on the nature of parent-child interactions have received less explicit attention. Research strategies to adequately capture these complex transactions between parents and children are elusive (Sameroff \& MacKenzie, 2003). Nevertheless, child variables, such as the use of prelinguistic communicative gestures (Olson \& Masur, 2013; Wu \& Gros-Louis, 2015) and children's sensory hyper- and hypo-reactivity (Kinard et al., 2016) have been implicated in influencing the frequency and type of parent responses occurring during parent-child interactions. In the context of the present study, it is important to consider the potential complexities of the transactions between parents and their children with ASD when children may engage in fewer behaviors that elicit parental responses or engage in unusual behaviors that may alter the quality of parent responses, while at the same time, some parents may have subclinical symptoms of ASD that influence their perceptions of and reactions to their children's behaviors. This study represents an initial effort to examine some of these interrelationships among parental BAP characteristics, parental verbal responsiveness, and child behaviors in the context of parent-child interactions involving mothers versus fathers.

The long-term goal of this program of research is to develop an individualized, parentimplemented, social communication intervention for young children with ASD that effectively involves both fathers and mothers. The aims of the current study were to investigate several unanswered questions regarding relationships between parent responsiveness and BAP characteristics and the language skills of children with ASD. Specifically, this investigation sought to identify any concurrent associations between parental BAP characteristics and child engagement and language skills, and to examine whether associations between parental BAP characteristics and child language remain significant after accounting for parental verbal responsiveness. We predicted that child engagement and language skills would be concurrently negatively associated with scores on the Broad Autism Phenotype Questionnaire (BAPQ), a measure of parental BAP characteristics. In addition, we predicted that concurrent negative relationships between parental scores on BAPQ subscale and child language skills would decrease with increased parent use of responsive verbal behaviors. 


\section{Methods}

\section{Participants}

Sixteen children with ASD (12 boys and 4 girls) and their mothers and fathers participated in this study. Child participants met the following inclusion criteria: (a) chronologic age between 36 and 69 months; (b) existing clinical diagnosis of ASD, confirmed by the cutoff scores on the Autism Diagnostic Observation Schedule (ADOS; Lord, Rutter, DiLavore and Risi 2006); (c) no severe sensory or motor impairments; and (d) no identified metabolic, genetic, or progressive neurological disorders. In addition, each participating child was required to have two biological parents, or married caregivers, residing with the child continuously since birth. Table 1 provides demographic information for parents and children. Other findings from this sample have been reported previously (Flippin \& Watson, 2015).

\section{Standardized Measures}

The ADOS (Lord et al. 2006) is a standardized, semi-structured play-based assessment of social interaction, verbal and non-verbal communication, as well as stereotyped behaviors and restricted interests. The ADOS is comprised of four modules, based on a participant's particular expressive language level. ADOS Gotham algorithm scores (Gotham, Risi, Pickles and Lord. 2007) meeting cut-offs for ASD were an inclusion criteria for child participants in this study. Although each children in study had a previous community diagnosis of autism, the ADOS was administered by research-reliable project staff in order to confirm diagnosis of ASD and obtain severity scores for fifteen of the sixteen participating children. For one child, ADOS had recently been completed by the local agency determining Part $\mathrm{C}$ eligibility and scores were shared with study, however the ADOS was not administered by project staff.

Calibrated severity scores, which allow comparisons across different ADOS modules, were calculated from the ADOS total scores according to Gotham, Pickles, and Lord (2009). Calibrated severity scores range from 1 to 10 , with scores from 6 to 10 corresponding to approximate fifths of individuals included in Gotham et al. who received ADOS algorithm scores classifying them in the autism range. Severity scores are reported for children in our sample for descriptive purposes (see Table 2).

The MSEL (Mullen, 1995) is a comprehensive measure of cognitive function for children from birth to 68 months of age. The test is comprised of five subscales: gross motor, visual reception, fine motor, receptive language, and expressive language. The MSEL is recommended for evaluating verbal and non-verbal development quotients for young children with autism (Akshoomoff, 2006). The Visual Reception (VR) subscale measures visual processing skills, spatial organization, and visual memory. The MSEL VR subscale has been shown to be a predictor of later receptive and expressive language growth in children with ASD (Bedford, Pickles \& Lord, 2016). For participating children, scores obtained within 3 months were shared from larger projects, or the VR subtest was administered concurrent with their participation in this study (for three children). Data for VR T-scores (mean of 50, SD =10) are provided for descriptive purposes (see Table 2); 
however, the summary statistics include seven (of 16) participating children who achieved the lowest possible T-score on the VR subtest (i.e., 20).

The Preschool Language Scale-4 (PLS-4; Zimmerman et al., 2002) was administered to assess child language skills. The PLS-4 is a standardized measure of receptive and expressive language skills for children birth to 6 years, 11 months of age. The test is composed of two subscales: Auditory Comprehension and Expressive Communication. The PLS-4 yields norm-referenced scores for each subscale, as a well as a total score computed from the two subscales. PLS-4 standard scores have a mean of 100 and a standard deviation of 15 . For analytic purposes, total language raw scores were used as the metric of child language skills, as 5 of 16 children in this study obtained the lowest possible total language standard score (i.e., 50) on the PLS-4 (see Table 2).

The Broad Autism Phenotype Questionnaire (BAPQ; Hurley et al. 2007) was administered to assess personality and language characteristics of the BAP with participating parents. The BAPQ was chosen because it is an efficient and valid informant report instrument designed to identify and characterize the BAP in adult relatives of individuals with ASD. To avoid potential response bias, authors of BAPQ entitled the form distributed to parents "Personality Styles and Preferences Questionnaire." The questionnaire is composed of 36 items, organized into 3 subscales (Aloof; Rigid; Pragmatic Language) that reflect the social, stereotyped repetitive, and communication behavioral domains characteristic of autism spectrum disorder. Items are rated along a six point Likert scale (ranging from "very rarely" to "very often"). Each mother and father enrolled in the study completed the BAPQ twice: once as a self-report questionnaire and once as an informant questionnaire for his/her spouse. Composite "best estimate" scores were then calculated by averaging corresponding self- and informant-report values for each of the individual subscales as well as Total BAPQ score, with higher scores indicative of the BAP. Table 3 describes BAPQ scores for participating parents. Sensitivity and specificity of composite scores are $81.8 \%$ and $78.1 \%$ respectively (Hurley et al., 2007).

Although the current study included only 16 mother-father dyads, a wide range of BAP characteristics were represented within the parent sample. Among individual parents, three mothers and five fathers did not meet criterion on any of the BAPQ subscales (Aloof, Rigid, Pragmatic Language); whereas, two mothers and three fathers met criterion on all 3 subscales. In addition to the wide range of BAPQ scores for individual mothers and fathers, there was also a wide range of combinations of BAP characteristics within and across parent couples. For example, at one extreme, two mother-father dyads did not meet criterion for any of the three BAPQ subscales. At the other extreme, for one parent dyad, the mother met criteria for all three BAPQ subscales whereas the father met for none. Table 4 describes the various BAP characteristics of the 16 parent couples.

Participating parents completed a demographic questionnaire to indicate ethnicity (Hispanic or non-Hispanic) and race (i.e., American Indian/Alaska Native; Asian; Black/AfricanAmerican; Native Hawaiian/Pacific Islander; White), and to measure levels of household income and parental education. Household income was measured as one of six levels: (a) less than $\$ 20,000$; (b) $\$ 20,000-\$ 39,999$; (c) $\$ 40,000-\$ 59,999$; (d) $\$ 60,000-\$ 79,999$; (e) 
$\$ 80,000-\$ 99,999$; (f) greater than $\$ 100,000$. Parental education was measured as one of six highest levels of education completed: (a) grades 1-11; (b) high school graduate/GED; (c) Associates/Technical degree; (d) Bachelors; (e) Masters; or (f) Doctorate/equivalent professional level degree.

\section{Observational Measures}

Parent-child Naturalistic Play Observations-Mother-child and father-child fifteenminute naturalistic play observations were conducted in the lab. The order of mother-child and father-child sessions was counterbalanced across parents to control for order effects. Two different standard, parallel sets of age-appropriate toys were assembled so that toys would be equally novel to each child in play sessions with mothers and with fathers. Each toy set included masculine (male figures, trucks, cars), feminine (female figures, baby dolls) and gender-neutral (blocks, twirlers) categories. At the start of each session, parents were directed to, "Play as you would normally play at home. Feel free to use some or all of the toys. We ask that if you are going to sit, parents sit in the larger blue chair so that it is easier to see the child on video." Sessions were video-recorded for later coding.

Coding and Reliability-Observational measures in this study were adapted from a coding schema developed by Yoder, Fey, Thompson, McDuffie, and Lieberman (2007). Using Procoder software (Tapp, 2003), fifteen minutes of each parent-child play session were coded at 5 -second intervals (180 intervals). Coding was conducted in three passes.

Codeable: On the first pass, coders determined whether each 5-second interval was either (a) codeable or (b) uncodeable. Codeable intervals required the child to be visible on screen for the entire 5-second interval period. For all codeable intervals, child engagement was then coded on the second pass through the media files.

Child engagement: On the second pass, child-initiated engagement was coded for all intervals in which the child either looked at or physically touched an object or person. Childinitiated engagement in this study did not require that the child initiate an intentional communication bid to the parent. Rather, the child merely needed to initiate attention to or physical engagement with an object or event in such a way that a parent can determine the child's focus of attention. Specifically, three types of child-initated engagement were coded: "look engagement"; "touch engagement"; or "no engagement". If a child looked at a toy, person, or event during the 5-second interval, an instance of visual or "look" engagement was coded. If a child actively touched a toy during the 5-second interval, tactile or "touch" engagement was coded. Touch engagement was not coded if a child was engaged in perseverative or self-stimulatory use of an object. For intervals in which a child both actively touched one toy, but looked at another, the child was credited with visual engagement. If a child was not engaged with either looking at or touching a person or object (e.g., child was running, climbing) during the duration of the 5-second interval "no engagement" was coded. In addition, if a parent redirected a child's attention to a new focus during a given interval, the child was only credited with child-initiated engagement if the child maintained a focus on the object introduced by the parent (by looking at and/or touching it) over the following two 5-second intervals. Thus, parents who redirected their children's attention frequently 
reduced the number of intervals in which a child could be scored as showing child-initiated visual or tactile engagement.

Parent Verbal Responsiveness: On the third pass through the media files, parent responses were coded for each 5-second interval for which a child was coded with visual or tactile engagement on the previous pass. Parent utterances (i.e., comments and requests) were coded as responsive if they (a) referenced the child's focus of attention (e.g., object or event), and (b) had a specific semantic relationship to the child's focus of attention, including the object, proprieties of the object (e.g., color, size, textures, sounds associated with the object) and action, or qualities of the action (e.g., "black hat"; "Can you put the hat on top?"). Parent utterances that referenced an object or action outside of the child's focus of attention were not coded as responsive. In addition, parent use of affirmatives (e.g., "all right"; "good job"), negative response words, interjections and vocatives (e.g., "um," "Eh?”), and routinized utterances, (e.g., counting, alphabet recitation, songs, finger plays) were not coded as parent verbal responsiveness.

Reliability: Coding of parent-child observations videos was completed by two coders, with backgrounds in public health, and communication sciences and disorders, respectively. The primary coder was blind to the research questions and hypotheses, and both coders were trained to $80 \%$ reliability on each behavioral code on practice videos. The second coder independently coded the full parent-child play sample and then codes were compared. Intraclass correlations (ICCs) were calculated across a randomly selected $25 \%$ of the parentchild play observations (four mother-child sessions and four father-child sessions). ICC values were uniformly above .80 for each coded variable, exceeding the ICC value of .70 suggested to reflect acceptable reliability (Mitchell 1979). ICCs were as follows: codeable, . 99; 95\% CI [0.88, 1.00]; touch lead, 1.00; 95\% CI = [0.99, 1.00]; look lead, .96; 95\% CI [0.96, 0.99]; mother verbal responsiveness, .99; $95 \%$ CI [0.96, 1.00]; father verbal responsiveness, $.99 ; 95 \% \mathrm{CI}[0.86,1.00]$.

\section{Procedures}

For child participants recruited from larger projects $(n=13)$, the PLS-4, parent-child play observations, and parent questionnaires were completed on the same day. For two children not recruited from larger studies, MSEL VR subscale and ADOS were administered by research-reliable project staff prior to PLS-4, and parent-child play observations and questionnaires. Finally for one child for whom the ADOS was administered by local agency determining Part C eligibility and shared with this study, the MSEL VR subscale was completed prior to PLS-4, parent-child play observations, and questionnaires. Table 2 describes child assessment data. Sessions were scheduled on weeknights and Saturdays, as needed, to allow both fathers and mothers to participate. Upon completion of the assessments and observations, each participating family received $\$ 25$ for travel expenses.

\section{Plan for Data Analyses}

Prior to answering the research questions, distributions of variables were examined to determine whether they met assumptions of normality. Child scores on the MSEL VR 
subscale and PLS-4 total language raw scores were negatively skewed; however, ADOS Gotham algorithm scores (Gotham et al., 2009) were more normally distributed, suggesting the study sample reflected a wide range of ASD characteristics. Next, associations between levels of household income and parent education and measures of child language skills and parent verbal responsiveness were examined. Levels of household income were not significantly associated with the child MSEL VR subscale ( $r h o=-.123, p=.313$ ) or PLS-4 scores $(r h o=-.163, p=.273)$ and therefore were not considered further. To answer the three research questions, correlations were run between: (1) parent BAPQ subscale scores and parent responsiveness; (2) parent BAPQ subscale scores and child language raw scores; and (3) parent BAPQ scores and child language raw scores, controlling for parent responsiveness. For all correlations, non-parametric Spearman rho was utilized. The Spearman correlation coefficient is based on the ranked values for each variable rather than the raw data and is appropriate for examining monotonic relationships between two variables, in which the variables tend to change together, but not necessarily at a constant rate. Statistical analyses were conducted using SPSS 24.0 for Mac. Results of data analysis procedures are described relative to each of the research questions below.

\section{Results}

\section{Research question 1. Are there concurrent associations between parent BAP characteristics and frequency of child-initiated engagement?}

Spearman's rho correlations were run to examine relationships between frequency of childinitiated engagement and parent BAPQ scores. Child-initiated engagement was negatively associated with mothers' scores on both Aloof ( $r h o=-.586, p=.01)$ and Rigid BAPQ subscales $(r h o=-.568, p=.02)$. In contrast, child-initiated engagement was not associated with mothers' Pragmatic Language subscale scores ( $r h o=-.225, p=.40)$. Therefore, as the frequency of child-initiated engagement increased, mothers tended to show less aloof and rigid characteristics, as indicted by lower BAPQ subscale scores. For fathers, child-initiated engagement was also not associated with paternal Aloof ( $r h o=.049, p=.42)$ or Rigid BAPQ subscale scores $(r h o=.089, p=.37$ ); however, a positive association was found between frequency of child-initiated engagement and fathers' scores on the Pragmatic Language subscale ( $r h o=.470, p=.03$ ), indicating children showed more engagement during interaction with fathers with more impacted pragmatic language skills.

\section{Research question 2. Are there concurrent associations between parent BAP characteristics and verbal responsiveness?}

To answer the second research question, Spearman's rho correlations were run to examine the concurrent relationships between parent verbal responsiveness and mothers' and fathers' BAPQ subscale scores. For mothers, significant negative associations were found between frequency of responsive verbal behaviors and Aloof subscale scores ( $r h o=-.621, p=.01$ ), indicating that mothers who had higher Aloof subscale scores tended to use fewer responsive verbal behaviors in interactions with their children with ASD. In contrast, mothers' scores on the Rigid and Pragmatic Language subscales were not significantly associated with verbal responsiveness ( $r h o=-.375, p=.152 ; r h o=-.285, p=.142$, respectively), although the negative correlations were in the predicted direction. For fathers, no significant 
associations were found between frequency of responsive verbal behaviors and subscale scores of the BAPQ: Aloof $(r h o=-.080, p=.365)$; Rigid ( $r h o=.181, p=.251)$; or Pragmatic Language ( $r h o=.293, p=.136$ ). The directions of these nonsignificant associations also varied (Table 5).

\section{Research question 3. Are there concurrent associations between parent BAP characteristics and child language skills and do these relationships remain significant after accounting for parent verbal responsiveness?}

For question 3, we first ran Spearman's rho correlations to examine concurrent relationships between child language skills and parent BAPQ scores. For mothers, significant negative associations were found between child language skills and parent scores on Aloof ( $r h o=-$. $500, p=.048)$ and Rigid BAPQ subscales ( $r h o=-.691, p=.003$ ), indicating that higher maternal scores on the Aloof and Rigid subscales were concurrently associated with lower child language scores. In contrast, mothers' scores on the Pragmatic Language subscale were not associated with child language skills ( $r h o=-.285, p=.284$ ). For fathers, no associations were found between child language skills and paternal scores the three BAPQ subscales: Aloof ( $r h o=-.013, p=.968)$; Rigid $(r h o=.186, p=.245)$; Pragmatic Language $($ rho $=.118, p=.331)($ Table 5$)$.

Given the significant associations between child language and mothers' Aloof and Rigid BAPQ subscales, we next ran partial correlations for mothers' Aloof and Rigid BAPQ subscales to examine whether associations between child language skills and maternal scores on these two BAPQ subscales remained significant after controlling for frequency of mothers' responsive verbal behaviors. After partialling out maternal responsive verbal behaviors, correlations between mothers' scores on the Aloof subscale and child language scores were no longer significant ( $r h o=.33, p=.22$ ), indicating that mothers use of responsive verbal behaviors is a potential mediator of the relationship between aloof parental BAP characteristics and child language skills. In contrast, correlations between mothers' scores on the Rigid BAPQ subscale and child language skills remained moderately strong and significant after partialling out mothers' verbal responsive behaviors $($ rho $=-.58, p=$. 022).

\section{Discussion}

In this study, we examined the extent to which parental BAP characteristics were concurrently associated with parents' verbal responsiveness to their young children with ASD, and with child-initiated engagement and level of language functioning. Several findings emerged.

\section{Parental BAP and Child-Initiated Engagement}

First, significant concurrent associations were found between frequency of child-initiated engagement and mothers' scores on the Aloof and Rigid subscales. Children initiated less engagement with mothers who had higher Aloof and Rigid BAPQ scores. This finding is consistent with findings from other recent studies documenting associations between maternal BAP traits and child social communication skills (Hasegawa et al., 2015; Taylor et 
al., 2013). In considering these findings however, it is important to remember that the operational definition of a child-initiated engagement in this study does not require that the child initiate an intentional communication bid to the parent. Rather, the child merely needs to have initiated attention to or engagement with an object or event in such a way that a parent can determine the child's focus of attention. Thus, it appears that mothers who have more aloof and rigid characteristics have children who tend to be less attentive to and engaged with their environment overall, and not just less socially engaged. As Hasegawa et al. (2015) and Taylor et al. (2013) describe, it is unclear whether associations such as these between parental BAP status and child-initiated engagement are caused by genetic or environmental factors, or a combination of both. For mothers, Pragmatic Language subscale scores were unrelated to child-initiated engagement.

In contrast to the findings for mothers, fathers' Aloof and Rigid subscale scores were unrelated to child-initiated engagement; however, a positive association was found between frequency of child-initiated engagement and fathers' scores on the Pragmatic Language subscale. That is, children in this study used more visual and tactile engagement with fathers whose pragmatic language characteristics more strongly resembled those seen in individuals with ASD. The finding of positive correlations between paternal scores on the Pragmatic Language subscale and child-initiated engagement was unexpected and warrants further study. There may be several explanations for why Pragmatic Language subscale scores were significantly correlated with child-initiated engagement for fathers and not for mothers (where the association was of a low magnitude as well as nonsignificant). One possible explanation is that items of the BAPQ Pragmatic Language subscale are specific to adultadult relationships and may not directly generalize to parent-child communication exchanges, where appropriate pragmatic performance may be easier. In fact, Pragmatic Language subscale items are marked in asterisk to prompt respondents to answer in terms of "casual interaction with acquaintances, rather than special relationships such as with close friends and family members" (e.g., "I can tell when someone is not interested in what I am saying"; "I am good at making small talk"; "I can tell when it is time to change topics in conversation"; Hurley et al., 2007, p. 1689). Another potential explanation may be that fathers with pragmatic language difficulties are less directive of child play, allowing their children to take the lead in play interactions. That is, the style used by these fathers may not have imposed limits on the number of intervals in which a child could be credited with initiating engagement. Finally, it may be the case that the language models of fathers are in some way supportive in interactions with children who share pragmatic language deficits.

\section{Parent Verbal Responsiveness and Child Language}

Similar to findings with child-initiated engagement, mothers' scores on the Aloof and Rigid BAPQ subscales were also negatively related to child language scores. In contrast, none of the paternal BAPQ subscale scores were associated with child language scores. Findings of significant correlations between parental BAP traits and child language skills with mothers but not fathers in the current study are consistent with results of Hasegawa et al. (2015). In their study, higher Autism Spectrum Quotient (Baron-Cohen, Wheelwright, Skinner, Martin \& Clubley, 2001) scores for mothers, but not fathers, were a significant predictor of higher Social Responsiveness Scale (Constantino, 2002) total scores in children. Findings of 
significant associations between BAP child language skills and maternal, but not paternal, characteristics may reflect the stronger history of interaction between the participating mothers and children with ASD, as 14 of the 16 mothers in this study identified caregiving as their primary role. In addition, effects may be operating across parent couples. For instance, higher maternal scores on the Aloof subscale were correlated with less childinitiated engagement during mother-child interactions only, whereas higher maternal scores on the Rigid subscale were associated with less child-initiated engagement across both mother-child and father-child interactions. Thus it is possible that mothers and fathers may be taking cues from one another in communicating with their child with ASD. For example, a parent with higher Rigid subscale scores may influence the parent partner to adopt a less flexible parent-child communication and interaction style. Alternatively, a parent with higher Aloof subscale scores may use communication styles related to the Aloof subscale (e.g., using long pauses while talking, losing track of the conversation topic, not being "in tune" with another person during conversation) that are less influential on the partner's parentchild communication style. The potential impact of couple effects is interesting to consider, and future research with larger samples is warranted to determine whether this relationship between the frequency of child-initiated engagement and parent BAPQ subscale scores and is found with a larger sample and persists over time.

\section{Parent Verbal Responsiveness as Potential Mediator for Parental BAP and Child Language Skills}

After controlling for mothers' responsive verbal behaviors, negative correlations between mothers' scores on the Aloof subscale and child language scores were no longer significant, suggesting that increased frequency of responsive parent verbal behavior potentially mediates the relationship between aloof traits and child communication skills. However, such interpretations based on examining concurrent associations are speculative at this stage. As we have reported elsewhere, there are concurrent positive correlations between the frequency of parent verbal responses and child language skills in this sample for both mothers and fathers (BLINDED). Given evidence supporting bidirectional influences between child language level and parent responsiveness (e.g., Sterling, Warren, Brady \& Fleming, 2013; Warlaumont, Richards, Gilkerson \& Oller, 2014; Yoder et al., 2015), we acknowledge that longitudinal and experimental studies could better elucidate the potentially complex relationships among parent BAPQ characteristics, child language, and parent verbal responsiveness.

Whereas partialling out maternal verbal responsiveness resulted in a nonsignificant correlation between the Aloof BAPQ subscale scores and child language, the negative correlation between maternal Rigid BAPQ subscale scores and child language scores remained substantive and significant after controlling for mothers' use of responsive verbal behaviors. In this study, parent utterances were coded as responsive if they (a) referenced the child's focus of attention (e.g., object or event), and (b) had a specific semantic relationship to the child's focus of attention, including the object, proprieties of the object (e.g., color, size, textures, sounds associated with the object), and action, or qualities of the action. However, flexibility of the verbal response was not measured in this coding schema. Parent language was coded as responsive even if parents repeated the same or similar responses to a 
child's continued visual or tactile engagement related to the same target. Thus, simply increasing frequency of verbal responses may not mediate the negative relationship between parental rigid traits and child communication skills, especially if verbal responses of mothers with high scores on the Rigid BAPQ subscale reflect repetitiveness or inflexibility in the way mothers are responding to their children.

\section{Study Limitations}

Results from this study have provided some early evidence regarding concurrent associations between the language skills of children with ASD and parent verbal responsiveness and BAP characteristics; however, several limitations must be highlighted. The first limitation of this study is the small sample size $(n=16)$, which restricted our choices of appropriate statistical models, the ability to rule out potential confounders, or adjust for the multiple correlation coefficients examined without greatly inflating the risk of Type II errors. Second, this study examined relationships between parent BAPQ scores, verbal responsiveness, and child language skills at a single time point. Other research designs that examine longitudinal associations and potentially manipulate variables of interest (e.g., increasing parent responsiveness through coaching) are necessary to determine whether these associations hold up across time, examine temporal precedence, and test causal hypotheses. Third, although the parent sample varied racially and ethnically, median family income was $\$ 100,000$, and the middle-to-high socio-economic status of the sample may have affected results. Fourth, parents who participated in this study may differ in important ways from parents who do not choose to participate in research. Finally, due to the nature of the research questions, parents who participated in this study were married, which is not reflective of all families of children with ASD.

\section{Clinical Implications and Future Directions}

An emerging body of evidence supports the inclusion of pre-treatment child factors (e.g., verbal skills, joint attention skills, imitation skills) as potentially influential variables on intervention efficacy for children with ASD. In contrast, few studies have examined the potential influence of pre-treatment parent factors on communication outcomes for children with ASD. However, parents who demonstrate characteristics of the BAP (i.e., aloofness, rigid personality, pragmatic language problems) may benefit from coaching models that are tailored to their unique social communication needs, strengths, and personalities. Individualizing parent coaching to fit parent pre-intervention characteristics in this way may increase the fidelity and effectiveness of parent-implemented intervention, resulting in improved social communication outcomes for children with ASD. Specifically, parents who meet criteria for Aloof characteristics of the BAP may benefit from interventions focused on increasing verbal responsiveness by coaching parents to share their child's focus of attention and model language that linguistically maps to the child's focus of attention. Conversely, coaching models focused solely on increasing frequency of responsive behaviors may be less effective for parents who meet criteria for rigid BAP characteristics. For these parents, coaching to help parents use a variety of flexible responsive verbal behaviors in order to better scaffold their children's engagement and language learning may be more effective. Overall, understanding which responsive strategies are both beneficial to children with ASD 
and amenable to parents may be an important component of developing effective parentimplemented intervention.

In summary, findings from this observational study provide important early empirical support for continued investigations to examine associations between pre-intervention parent variables and communication skills of children with ASD. More research is needed to examine the best set of parent and child predictors of language outcomes for children with ASD. Specifically, future intervention research should investigate whether individualized parent coaching fits the needs and strengths of parents who demonstrate characteristics of the BAP. Understanding which responsive strategies best fit both parent and child preintervention variables may be an important component in developing maximally effective social communication intervention for children with ASD. Identifying these variables will allow for greater specificity in matching parent coaching strategies to pre-treatment child and parent variables and may be beneficial for long-term communication outcomes.

\section{Acknowledgments}

Funding.

Funding was provided by National Institute on Deafness and Other Communication Disorders (NIDCDR01DC006893) and Institute of Education Sciences (R324B070056).

\section{References}

Akshoomoff N. Autism spectrum disorders. Child Neuropsychology. 2006; 12:245-246.

American Psychological Association. Diagnostic and statistical manual of mental disorders (DSM-V). 5th. Washington, DC: Author; 2013.

Baker CE. Fathers' and mothers' home literacy involvement and children's cognitive and social emotional development: implications for family literacy programs. Applied Developmental Sciences. 2013; 17:184-97.

Baker CE, Vernon-Feagans L, the Family Life Project Investigators. Fathers' language input during shared book activities: Links to children's kindergarten achievement. Journal of Applied Developmental Psychology. 2015; 36:53-59.

Baron-Cohen S, Wheelwright S, Skinner R, Martin J, Clubley E. The Autism-Spectrum quotient (AQ): Evidence from Asperger syndrome/high-functioning autism, males and females, scientists and mathematicians. Journal of Autism and Developmental Disorders. 2001; 31:5-17. [PubMed: 11439754]

Bedford R, Pickles A, Lord C. Early gross motor skills predict the subsequent development of language in children with autism spectrum disorder. Autism Research. 2016; 9:993-1000. [PubMed: 26692550]

Bernstein Ratner N. Patterns of parental vocabulary selection in speech to young children. Journal of Child Language. 1988; 15:481-492. [PubMed: 3198717]

Bishop DV, Maybery M, Maley A, Wong D, Hill W, Hallmayer J. Using self-report to identify the broad phenotype in parents of children with autistic spectrum disorders: A study using the AutismSpectrum Quotient. Journal of Child Psychology and Psychiatry. 2004; 45:1431-6. [PubMed: 15482503]

Carter AS, Messinger DS, Stone WL, Celimni S, Nahmias AS, Yoder P. A randomized controlled trial of Hanen's "More Than Words" in toddlers with early autism symptoms. Journal of Child Psychology and Psychiatry. 2011; 52:741-52. [PubMed: 21418212]

Constantino, JN. The Social Responsiveness Scale. Los Angeles, CA: Western Psychological Services; 2002. 
Flippin M, Watson LR. Fathers' and mothers' verbal responsiveness and the language skills of children with autism spectrum disorder. American Journal of Speech-Language Pathology. 2015; 24:400410. [PubMed: 25836377]

Flippin M, Watson LR. Fathers' and mothers' verbal responsiveness and the language skills of children with autism spectrum disorder. American Journal of Speech-Language Pathology. 2015; 24:400410. [PubMed: 25836377]

Folstein S, Rutter M. Infantile autism: A genetic study of 21 twin pairs. Journal of Child Psychology and Psychiatry. 1977; 18:297-321. [PubMed: 562353]

Freedman BH, Kalb LG, Zablotsky B, Stuart EA. Relationship status among parents of children with autism spectrum disorders: a population-based study. Journal of Autism and Developmental Disorders. 2012; 42:539-548. [PubMed: 21590433]

Gleason, JB. Fathers and other strangers: Men's speech to young children. In: Dato, D., editor. Developmental psycholinguistics: Theory and application, Georgetown University Roundtable on language and linguistics. Washington, DC: Georgetown University Press; 1975. p. 289-297.

Gotham K, Pickles A, Lord C. Standardizing ADOS scores for a measure of severity in autism spectrum disorders. Journal of Autism and Developmental Disorders. 2009; 39:693-705. [PubMed: 19082876]

Gotham K, Risi S, Pickles A, Lord C. The Autism Diagnostic Observation Schedule: Revised algorithms for improved diagnostic validity. Journal of Autism and Developmental Disorders. 2007; 37:613-627. [PubMed: 17180459]

Green J, Charman T, Pickles A, Wan MW, Elsabbagh M, Slonims V, the BASIS team. Parent-mediated intervention versus no intervention for infants at high risk of autism: A parallel, single-blind, randomised trial. Lancet Psychiatry. 2015; 2:133-140. [PubMed: 26359749]

Haebig E, McDuffie A, Ellis Weismer SE. The contribution of two categories of parent verbal responsiveness to later language for toddlers and preschoolers on the autism spectrum. American Journal of Speech Language Pathology. 2013; 22:54-70.

Hartley SL, Papp LM, Blumenstock SM, Floyd F, Goetz GL. The effect of daily challenges in children with autism on parents' couple problem-solving interactions. Journal of Family Psychology. 2016; 30:732-742. [PubMed: 27336179]

Hasegawa C, Kikuchi M, Yoshimura Y, Hiraishi H, Muenesue T, Nakatani Hideo, et al. Broader autism phenotype in mothers predicts social responsiveness in young children with autism spectrum disorders. Psychiatry and Clinical Neurosciences. 2015; 69:136-144. [PubMed: 24902617]

Hurley RS, Losh M, Parlier M, Reznick JS, Piven J. The broad autism phenotype questionnaire. Journal of Autism and Developmental Disorders. 2007; 37:1679-90. [PubMed: 17146701]

Kinard JL, Sideris J, Watson LR, Baranek GT, Crais ER, Wakeford L, Turner-Brown L. Predictors of parent responsiveness to 1-year-olds at-risk for autism spectrum disorder. Journal of Autism and Developmental Disorders. 2017; 47:172-186. [PubMed: 27785592]

Konstantareas MM, Mandel L, Homatidis S. The language patterns mothers and fathers employ with their autistic boys and girls. Applied Psycholinguistics. 1988; 9:403-414.

Landry SH, Smith KE, Swank PR. Responsive parenting: Establishing early foundations for social, communication, and independent problem-solving skills. Developmental Psychology. 2006; 42:627-642. [PubMed: 16802896]

Leech KA, Salo VC, Rowe ML, Cabrera NJ. Father input and child vocabulary development: The importance of wh-questions and clarification requests. Seminars in Speech and Language. 2013; 34:249-259. [PubMed: 24297617]

Lord, C., Rutter, M., DiLavore, P., Risi, S. Autism Diagnostic Observation Schedule. San Antonio, TX: Western Psychological Services; 2006.

Masur E, Gleason JB. Parent-child interaction and acquisition of lexical information during play. Developmental Psychology. 1980; 16:404-409.

McLaughlin B, Schultz C, White D. Parental speech to five-year-old children in a game-playing situation. Child Development. 1980; 51:580-582.

McDuffie A, Yoder P. Types of parent verbal responsiveness that predict language in young children with autism spectrum disorder. Journal of Speech, Language and Hearing Research. 2010; 53:1026-1039. 
Mitchell SK. Interobserver agreement, reliability, and generalizability of data collected in observational studies. Psychological Bulletin. 1979; 86:376-390.

Mullen, EM. Mullen Scales of Early Learning. Circle Pines, MN: American Guidance Service; 1995.

Olson J, Masur EF. Mothers respond differently to infants' gestural versus nongestural communicative bids. First Language. 2013; 33:372-387.

Pancsofar N, Vernon-Feagans L. Mother and father language input to young children: Contributions to later language development. Journal of Applied Developmental Psychology. 2006; 27:571-587.

Pancsofar N, Vernon-Feagans L, The Family Life Project Investigators. Fathers' early contributions to children's language development in families from low-income rural communities. Early Childhood Research Quarterly. 2010; 25:450-463. [PubMed: 21057648]

Pellegrini A, Brody G, Stoneman Z. Children's conversational competence with their parents. Discourse Processes. 1987; 10:93-106.

Pickles A, Starr E, Kazak S, Bolton P, Papanikolaou K, Bailey A, et al. Variable expression of the autism broader phenotype: Findings from extended pedigrees. Journal of Child Psychology and Psychiatry. 2002; 41:491-502.

Potter C. "It's the most important thing-I mean, the schooling": Father involvement in the education of children with autism. European Journal of Special Needs Education. 2016; 31:489-505.

Piven J, Palmer P, Jacobi D, Childress D, Arndt S. Broader autism phenotype: Evidence from a family history study of multiple-incidence autism families. American Journal of Psychiatry. 1997; 154:185-190. [PubMed: 9016266]

Rondal JA. Fathers' and mothers' speech in early language development. Journal of Child Language. 1980; 7:353-369. [PubMed: 7410499]

Rogers SJ, Estes A, Lord C, Vismara L, Winter J, Fitzpatrick A, et al. Effects of a brief Early Start Denver model (ESDM)-based parent intervention on toddlers at risk for autism spectrum disorders: a randomized controlled trial. Journal of the American Academy of Child and Adolescent Psychiatry. 2012; 51:1052-65. [PubMed: 23021480]

Rowe ML, Coker D, Pan BA. A comparison of fathers' and mothers' talk to toddlers in low-income families. Social Development. 2004; 13:278-91.

Ruser TF, Arin D, Dowd M, Putnam S, Winklosky B, Rosen-Sheidley B, Piven J, Tomblin B, TagerFlusberg H, Folstein S. Communicative competence in parents of children with autism and parents of children with specific language impairment. Journal of Autism and Developmental Disorders. 2007; 37:1323-1336. [PubMed: 17180460]

Sameroff AJ, MacKenzie MJ. Research strategies for capturing transactional models of development: the limits of the possible. Developmental Psychopathology. 2003; 15:613-640.

Scheeren AM, Stauder JEA. Broader autism phenotypes in parents of autistic children: Reality or myth? Journal of Autism and Developmental Disorders. 2008; 38:276-287. [PubMed: 17588199]

Schertz HH, Odom SL, Baggett KM, Sideris JH. Effects of joint attention mediated learning for toddlers with autism spectrum disorders: An initial randomized controlled study. Early Childhood Research Quarterly. 2013; 28:249-258.

Shannon JD, Tamis-LeMonda CS, London K, Cabrera N. Beyond rough and tumble: Low-income fathers' interactions and children's cognitive development at 24 months. Parenting: Science and Practice. 2002; 2:77-104.

Siller M, Hutman T, Sigman M. A parent-mediated intervention to increase responsive parental behaviors and child communication in children with ASD: A randomized clinical trial. Journal of Autism and Developmental Disorders. 2013; 43:540-555. [PubMed: 22825926]

Siller M, Sigman M. The behaviors of parents of children with autism predict the subsequent development of their children's communication. Journal of Autism and Developmental Disorders. 2002; 32:77-89. [PubMed: 12058846]

Siller M, Sigman M. Modeling longitudinal change in the language abilities of children with autism: Parent behaviors and child characteristics as predictors of change. Developmental Psychology. 2008; 44:1691-1704. [PubMed: 18999331]

Spiker D, Boyce G, Boyce L. Parent-child interactions when young children have disabilities. International Review of Research in Mental Retardation. 2002; 25:35-70. 
Sterling AM, Warren SF, Brady N, Fleming K. Influences on maternal responsivity in mothers of children with fragile X syndrome. American Journal of Intellectual and Developmental Disabilities. 2013; 118:310-326.

Tamis-LeMonda CS, Shannon JD, Cabrera NJ, Lamb ME. Fathers and mothers at play with their 2and 3-year-olds: contributions to language and cognitive development. Child Development. 2004; 75:1806-20. [PubMed: 15566381]

Tapp, J. Procoder for digital video: User manual. Nashville, TN: The John F. Kennedy Center, Vanderbilt University; 2003.

Taylor LJ, Mayberry MT, Wray J, Ravine D, Hunt A, Whitehouse AJO. Brief report: Do the nature of communication impairments in autism spectrum disorders relate to the broader autism phenotype in parents? Journal of Autism and Developmental Disorders. 2013; 43:2984-2989. [PubMed: 23619954]

Walker K, Armstrong L. Do mothers and fathers interact differently with their child or is it the situation which matters? Child: Care, Health, and Development. 1995; 21:161-181.

Warlaumont AS, Richards JA, Gilkerson J, Oller DK. A social feedback loop for speech development and its reduction in autism. Psychological Science. 2014; 25:1314-1324. [PubMed: 24840717]

Warren SF, Brady NC. The role of maternal responsivity in the development of children with intellectual disabilities. Mental Retardation and Developmental Disabilities. 2007; 13:330-338.

Wetherby A, Guthrie W, Woods J, Schatschneider C, Holland RD, Morgan L, Lord C. Parentimplemented social intervention for toddlers with autism: An RCT. Pediatrics. 2014; 134:10841093. [PubMed: 25367544]

Wolchik SA. Language patterns of parents of young autistic and normal children. Journal of Autism and Developmental Disorders. 1983; 13:167-180. [PubMed: 6863211]

Wu Z, Gros-Louis J. Caregivers provide more labeling responses to infants' pointing than to infants' object-directed vocalizations. Journal of Child Language. 2013; 42:538-561.

Yoder PJ, Fey M, Thompson T, McDuffie A, Lieberman RG. Partial interval time sampling of adaptive strategies for the Useful Speech Project. 2007 Unpublished manual.

Yoder PJ, Watson LR, Lambert W. Value-added predictors of expressive and receptive language growth in initially nonverbal preschoolers with autism spectrum disorders. Journal of Autism and Developmental Disorders. 2015; 45:1254-1270. [PubMed: 25344152]

Zimmerman, IL., Steiner, VG., Pond, RL. Preschool Language Scale-4. San Antonio, TX: The Psychological Corporation; 2004. 


\section{Table 1}

Demographic Information for Participating Families

\begin{tabular}{llll}
\hline & Mothers & Fathers & Children \\
\hline Age & (Years) & (Years) & (Months) \\
Mean & 38 & 39.6 & 53.3 \\
SD & 4.5 & 5.7 & 9.6 \\
Range & $30-47$ & $31-56$ & $40-69$ \\
Race/Ethnicity & & & \\
White/non-Hispanic & $69 \%$ & $63 \%$ & $56 \%$ \\
Hispanic & $12.5 \%$ & $18.75 \%$ & $0 \%$ \\
Asian & $18.75 \%$ & $18.75 \%$ & $18.75 \%$ \\
Mixed & $6.25 \%$ & $0 \%$ & $25 \%$ \\
Education & & & \\
Associates & $6.25 \%$ & $18.75 \%$ & \\
Bachelors & $18.75 \%$ & $18.75 \%$ & \\
Masters & $62.5 \%$ & $37.5 \%$ & \\
Doctorate/Professional & $12.5 \%$ & $25 \%$ & \\
Household Income & & \\
Median & $\$ 80,000-\$ 99,999$ & \\
Range & $\$ 20,000-\$ 39,999->\$ 100,000$
\end{tabular}




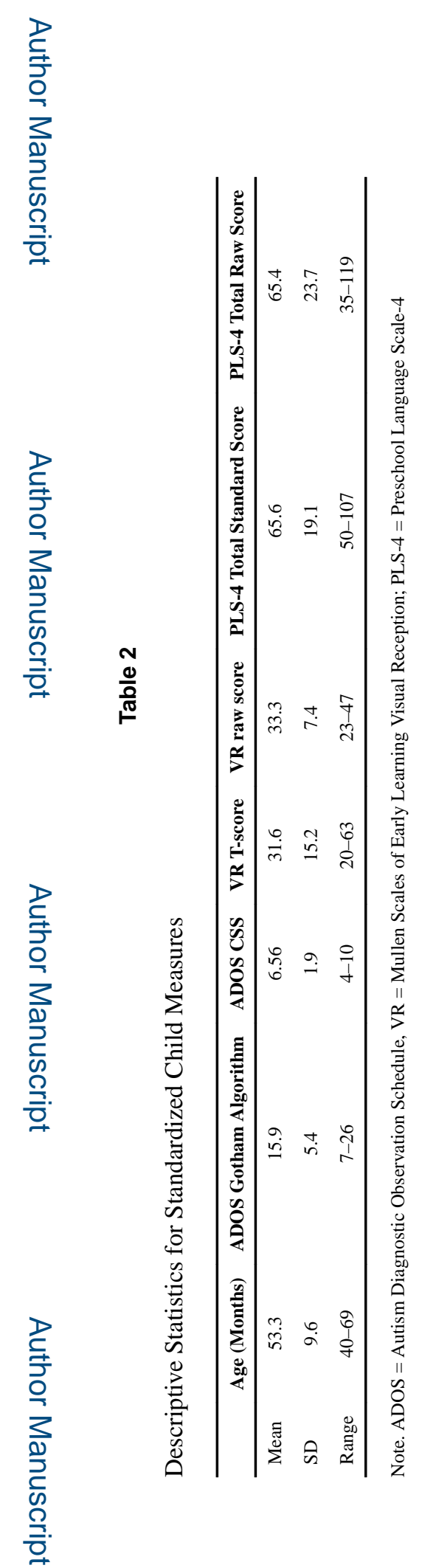



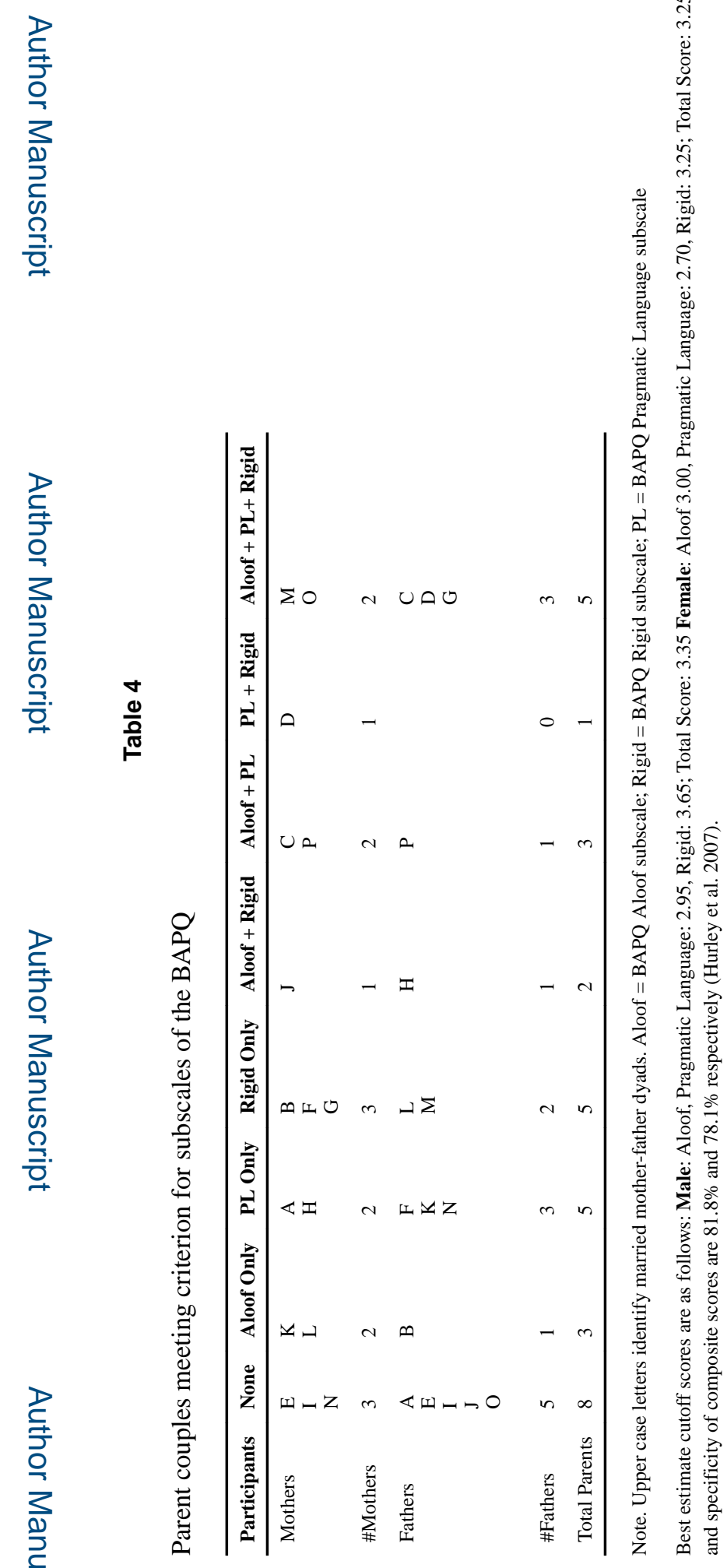

J Autism Dev Disord. Author manuscript; available in PMC 2019 June 01. 


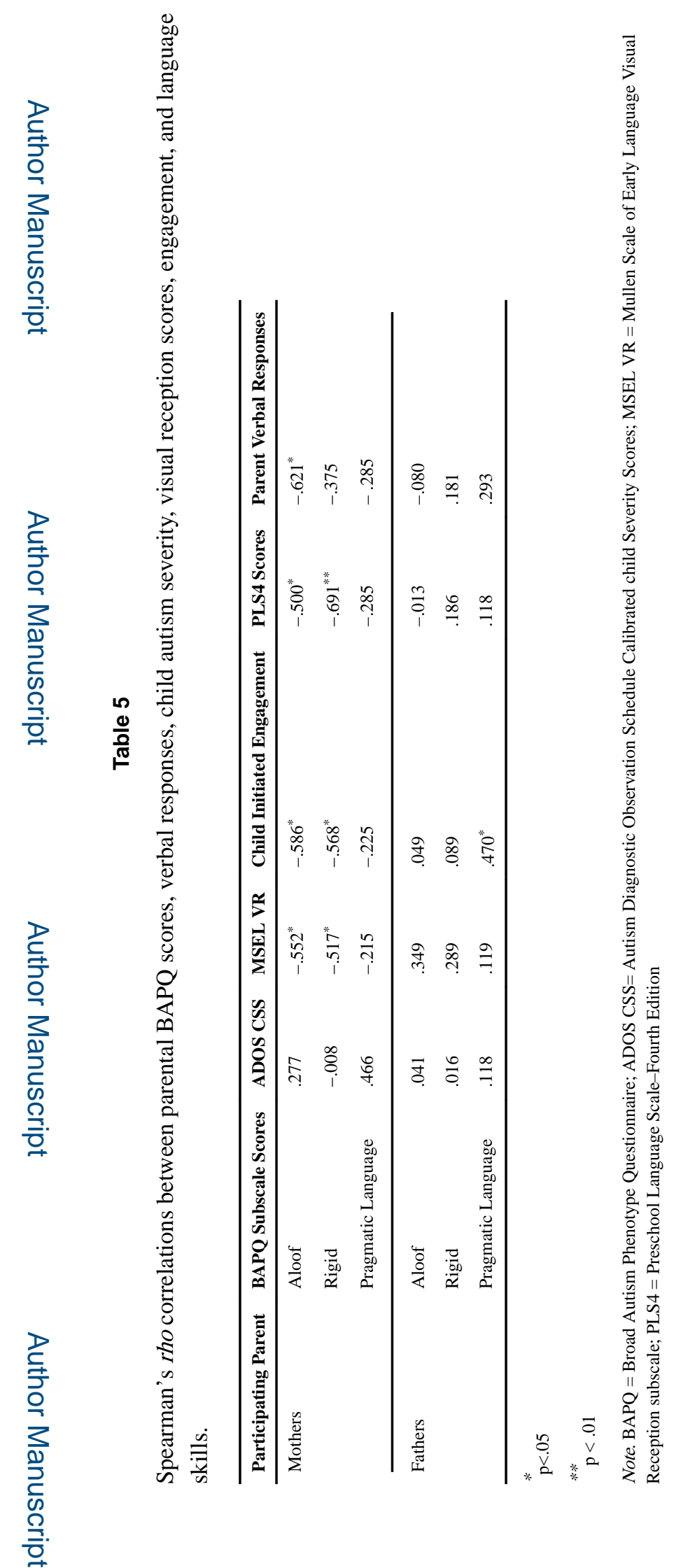

J Autism Dev Disord. Author manuscript; available in PMC 2019 June 01. 